\title{
Eficacia y eficiencia de las decisiones en entornos sistémicos complejos
}

\begin{abstract}
RESUMEN
En el estudio se identifica que la definición de situación problemática y la manera en que se enfrenta su solución depende del rol del decisor, que define la situación como problemas a resolver o problemas a demostrar (caso de negocio o caso de indagación o investigación), lo que facilita plantear la secuencia y forma de resolver el problema, considerando la eficacia y la eficiencia de la elección. Durante la etapa de contrastación de hipótesis, se consideran casos estudio a nivel de documento de trabajo, cuyo análisis permite corroborar la hipótesis de investigación, donde se observa que la perspectiva de la situación influye en el análisis y la búsqueda de la solución, además la posición del decisor y su rol dentro del campo de actuación que comprende la situación problemática, es relevante para la mejor elección.

Palabras clave: situación problemática, solución de problemas, eficacia decisional
\end{abstract}

EFICACIA Y EFICIENCIA de LAS DECISIONES EN ENTORNOS SISTÉMICOS COMPLEJOS

\section{ABSTRACT}

The study identifies the definition of problem situation and how it faces its solution depends on the role of the decision maker, that defines the situation as a problem to solve or demonstrate problems (business case or case of inquiry or investigation), it sequence which facilitates raising and how to solve the problem, considering the efficacy and efficiency of choice. During the stage of hypothesis testing, case studies are considered as working paper, their analyzes let corroborate the hypothesis of research, which shows that the perspective of situation influences analysis and search of solution, and it is corroborated that decision maker's position and role inside the field of action that covers problematic situation, is relevant to the best choice.

Keywords: problematic situation, troubleshooting, decisional effectiveness

\section{INTRODUCCIÓN}

El estudio se refiere a la toma de decisiones en entornos sistémicos, con elevada interacción de los componentes que intervienen y son afectados por la decisión. Estos entornos son los escenarios o campos de actuación donde se presentan las situaciones problemáticas, con diversos grados de complejidad, cuya percepción como problema a resolver depende de la posición y compromiso del decisor, en dicho escenario.

El objetivo general del presente estudio, es plantear la eficacia de la toma de decisiones donde la circunstancia comprende diversos escenarios de actuación, que comprenden las diferentes situaciones problemáticas, donde el decisor se ubica, asume posición y ejerce diversos roles.

Cada escenario plantea diferentes casos a resolver que se caracterizan por su creciente nivel de complejidad desde unidimensionales y simples hasta multidimensionales y complejos, desde el nivel operativo de manejo de recursos físicos hasta el nivel abstracto de conceptualización de modelos.

\section{PLANTEAMIENTO DEL ESTUDIO}

\subsection{Fundamentación del problema}

Entre los primeros antecedentes académicos se encuentra el método del caso de Harvard referido a la presentación de los hechos concretos y resolverlos bajo el criterio del pragmatismo por encima de las teorías y los conceptos abstractos, que es tomado por la metodología de la Ivey League para la enseñanza empresarial [9]. En los estudios de Peter Vail [15] se replanteó e integró el concepto sociotécnico con la visión de la ingeniería industrial [10]. Un concepto semejante es promovido por Eduardo Acuña en la Universidad de Chile mediante sus investigaciones acerca del efecto de las narraciones y la literatura en las decisiones y la gestión empresarial [3]. Se consideran diversos modelos de toma de decisiones, principalmente el modelo de decisión de Simons que sustenta las elecciones concatenadas y permanentes donde las elecciones no llevan necesariamente a la solución de los problemas [8].

* Doctor en Ingeniería Industrial, Magíster en Administración, Ingeniero Industrial, Economista. Profesor en la Facultad de Ingeniería Industrial - UNMSM. E-mail: aacevedo@speedy.com.pe, adolfo.acevedo@industrial.unmsm.pe

** Magíster en Dirección de Empresas, Ingeniero Industrial, estudios de Derecho. Profesora en la UPG de la Facultad de Ingeniería de Sistemas e Informática - UNMSM. E-mail: klinares@speedy.com.pe 
Además de la racionalidad científica que se sustenta en la inducción y reduccionismo para la solución de problemas, que se plasma en diferentes escuelas administrativas [6], se ha de considerar el enfoque holístico de las situaciones problemáticas, planteando las opciones con perspectiva sistémica [16]. Se complementa con los estudios del rol de gestión del ingeniero industrial [17].

\subsection{La pregunta de investigación}

Se plantea la siguiente interrogante de investigación:

¿Cómo es la toma de decisiones para la resolución de problemas, considerando la eficacia y eficiencia de la decisión?

Como problemas secundarios se plantean los siguientes:

a. ¿La complejidad del contexto influye en la eficacia de las decisiones?

b. ¿La posición y rol del decisor influye en la eficacia y eficiencia de las decisiones?

\subsection{Objetivos de la investigación}

El objetivo general es plantear el efecto de la circunstancia en la calidad de las decisiones en la resolución de problemas en empresa

Los objetivos específicos se refieren a definir los elementos que afectan la eficacia y eficiencia decisional en la resolución de problemas de empresa.

\section{LA METODOLOGÍA DE INVESTIGACIÓN}

El estudio es una investigación aplicada, que comprende una parte de investigación cualitativa y una parte de investigación cuantitativa, ya que por la naturaleza del problema, se requiere metodologías cualitativas y cuantitativas. Bajo este marco, las características epistemológicas del estudio se presentan en el Cuadro 1.

\subsection{Epistemología del estudio}

Para la investigación cualitativa se aplica el método hipotético-deductivo. A partir de los hechos se formulan las hipótesis preliminares y se plantea el modelo con sus variables, luego se generan las hipótesis del estudio que son contrastadas mediante la experimentación y análisis estadístico. Para la investigación cuantitativa se aplica el método inductivo-experimental, donde se recolectan datos por observación directa de los actores o decisores, los que se emplean para la contrastación de las hipótesis planteadas, mediante las herramientas de estadística inferencial y la experimentación [11]. Las características epistemológicas de la investigación se detallan en el Cuadro 1.

Para la validez metodológica del estudio, se consideran:

- Metodología de investigación en acción. La epistemología de investigación tiene el objetivo de ir mejorando la investigación a medida que se avanza en el mismo.

- Estadística inferencial y muestreo. Dentro de la categoría experimental pura que cumple con los requisitos de control y validez interna.

- Diseño de experimento en grupos aleatorios con instrumento preprueba y posprueba, más un grupo de control sin intervención experimental. cuya forma esquemática se muestra en el Cuadro 2.

Cuadro 1. Características epistemológicas del estudio

\begin{tabular}{|l|l|l|}
\hline \multicolumn{1}{|c|}{ CARACTERÍSTICAS } & \multicolumn{1}{c|}{ INV. CUALITATIVA } & \multicolumn{1}{c|}{ INV. CUANTITATIVA } \\
\hline Percepción de la realidad & 1. Subjetiva & 2. Objetiva \\
\hline Razonamiento & 3. Hipotético-deductivo & 4. Inductivo-experimental \\
\hline Finalidad & 5. Exploración & 6. Contrastación de hipótesis \\
\hline Perspectiva de investigación & 11. Racionalismo crítico & 12. Refutabilidad \\
\hline Validez metodológica & 19. Investigación en la acción & 20. Estadística inferencial y muestreo \\
\hline
\end{tabular}

Fuente: Elaboración propia. 
Cuadro 2. Diseño del estudio experimental

\begin{tabular}{|c|c|c|c|c|}
\hline Segmento & $\begin{array}{c}\text { Muestra } \\
\text { Grupo aleatorio }\end{array}$ & Elección preprueba & $\begin{array}{c}\text { Prueba de } \\
\text { experimento }\end{array}$ & Elección posprueba \\
\hline \multirow{2}{*}{ Aleatorio } & Ga1 & $\mathrm{O}_{1 \mathrm{~b}}$ & $\mathbf{X}$ & $\mathrm{O}_{2 \mathrm{~b}}$ \\
\cline { 2 - 5 } & $\mathrm{Ga} 2$ & $\mathrm{O}_{3 \mathrm{~b}}$ & -- & $\mathrm{O}_{4 \mathrm{~b}}$ \\
\hline
\end{tabular}

Fuente: Elaboración propia.

Donde:

$\mathbf{O}_{1 \mathrm{~b}}, \mathbf{O}_{\mathbf{2 b}}$ : Elecciones del decisor del grupo experimental, considerando posición y rol.

$\mathbf{O}_{3 \mathrm{~b}}, \mathbf{O}_{4 \mathrm{~b}}$ : Elecciones del decisor del grupo de control sin influencia experimental.

X $\quad$ Variable de experimento: posición y rol del decisor ante la situación problemática.

\subsection{Muestra, unidad de análisis y técnicas de recolección de datos}

La población comprende a los decisores en la gestión de organizaciones, sean empresas, instituciones y administración de gobierno. El tamaño de muestra se ha determinado mediante estadística inferencial para pequeñas muestras donde no se conoce la desviación estándar con un nivel de confianza de $95 \%$. De la población de decisores, se ha considerado una muestra total de 50 decisores, segmentados en dos grupos, de estudio y de control.

La unidad de análisis es el decisor en las organizaciones con nivel directivo o ejecutivo. Se considera como decisores a profesionales, académicos, egresados universitarios con grado académico superior (doctorado y maestría). La aplicación de la herramienta de recolección de datos, se realiza mediante talleres académicos, lo que configura muestras relacionadas, aunque definidas como muestra aleatoria, por cuanto provienen de diferentes organizaciones.

Para la recolección de datos se utilizan diversas técnicas, cuyos instrumentos de recolección de datos han sido validados mediante panel experto. Estas técnicas son las siguientes:

- Entrevistas. En la fase cualitativa del estudio se realizaron varias entrevistas abiertas y a profundidad con expertos, para identificar la perspectiva de cada experto respecto a los elementos relevantes de la eficacia de las decisiones.

- Encuestas. En la fase cuantitativa del estudio, se ha elaborado cuestionario de alternativa múltiple donde las variables, indicadores y eventos de decisión fueron validados con expertos en temas de decisiones y productividad.
- Investigación de gabinete. Se realizó el estudio de documentos sobre casos y decisiones, papers sobre técnicas racionales y heurísticas en resolución de problemas.

\section{MARCO TEÓRICO}

\subsection{Elementos de las dimensiones del mundo de la empresa}

La empresa se define por las dimensiones que delimitan sus fronteras y su accionar. Estas dimensiones son la estructura y el proceso que enmarcan el espacio de la poiesis o la tarea económica, que se define como la actividad productiva [2]

Para las adecuadas decisiones (direccionadas y correctas), el decisor se posiciona (mentalmente) en el campo poiético, considerando los cuatro elementos dimensionales del mundo organizacional [1], los que se interrelacionan e imbrican:

- Las estructuras físicas y mentales que representan el contexto para el proceso son el ambiente, recursos, activos [5], paradigmas, creencias y organización.

- Las fuerzas físicas y las fuerzas sociales como los propósitos, metas, planes, que ejercen presión interna y externa para la elección, transformación, movimiento y cambio.

- Las personas como fines o medios (decisores u operadores en la tarea productiva), aportan dirección, orientación y decisiones o proveen capacidad, conocimiento y motivación.

- Las actividades conceptuales y físicas, manuales e intelectuales, diseñadas y ejecutadas por las personas para alcanzar un producto de la mente humana o de la mano humana. 


\subsection{Las situaciones problemáticas dentro del espacio de la poiesis}

Toda situación problemática se encuentra inmersa dentro de un espacio de actuación [12]. No existen problemas aislados, existen situaciones problemáticas cuyas causas o efectos se intenta superar [4]. Existen problemas en toda situación problemática donde se busca pasar de un estado inicial indeseado $\mathbf{S}_{1}$ a una situación posterior deseada $\mathbf{S}_{2}$. Para el decisor, existe problema si y solo si, su elección y acción permite pasar de $\mathbf{S}_{1}$ hacia $\mathbf{S}_{2}$.

Las situaciones problemáticas se pueden diferenciar, sistémicamente, en seis tipos de problemas que se clasifican por el grado de dificultad en identificar y plantear la solución.

- SP1 - No existe problema, es narrativa, no se detecta situación indeseada o conflictiva por resolver. Posee propiedades de multidimensionalidad, multipropósito y multiniveles.

- SP2 - Problemas perversos, donde existen problemas que no se pueden resolver o no es posible lograr un resultado razonable en mediano plazo. Poseen propiedades emergentes impredecibles, son sistemas imbricados con múltiples causas y efectos [14].

- SP3 - Problemas evolutivos o cambiantes, son sistemas complejos con capacidad de evolucionar con el tiempo y adaptarse al entorno. Se caracterizan por la propiedad sistémica de la autopoiesis o generación de sí mismo.

- SP4 - Problemas complejos, son sistemas dinámicos, abiertos, conformados por componentes interconectados en múltiples niveles con capacidades sinérgicas Se organiza por tramas y redes sistémicas jerárquicas con efectos emergentes.
- SP5 - Problemas flexibles o blandos (soft), derivan de sistemas con diversos marcos de referencia (personas). Se caracteriza por la propiedad de la entropía donde la falencia de control va degradando los sistemas hasta su degeneración plena o desaparición.

- SP6 - Problemas rígidos duros (hard), derivan de sistemas con comportamiento previsible y reglas definidas y estables. Se caracterizan por la propiedad causal donde un input previsible conlleva un output determinado y planeado, por lo tanto, controlable.

\subsection{Situación problemática y problemas}

Para la resolución de problemas se requiere identificar el espacio de actuación, percibir la situación problemática e identificar el tipo de problema (situación sistémica) que se ha de resolver, para lo cual se han de enfocar las dimensiones a manejar, a partir de lo cual se seleccionan las variables verdaderamente importantes (Ver el Cuadro 3).

\section{EL ESTUDIO CUANTITATIVO DE LA EFICACIA DECISIONAL}

El desarrollo del estudio permitirá obtener elementos para determinar la eficacia de la elección ante variaciones en la circunstancia del decisor, específicamente, en la posición y rol que afectan el compromiso en la definición del problema y su consecuente solución.

\subsection{Planteamiento del estudio experimental}

Es necesaria la identificación de la influencia del contexto en la elección del decisor y si esto genera efectos complementarios en la eficacia de elegir y en la eficiencia o rapidez de respuesta para

Cuadro 3. Diseño del estudio experimental

\begin{tabular}{|c|l|c|}
\hline Clave & \multicolumn{1}{|c|}{ Tipo de problema } & $\begin{array}{c}\text { Situación } \\
\text { problemática }\end{array}$ \\
\hline A: & Situación problemática donde no existe problema. & SP1 \\
\hline B: & Situación problemática donde el problema es complejo, de difícil solución & SP2 \\
& o sin solución. & SP3 \\
\hline C: & Situación problemática donde existen varias soluciones. & SP4 \\
\hline D: & Situación problemática con solución única o estructurada. & SP6 \\
\hline
\end{tabular}

Fuente: Elaboración propia. 
encontrar la opción adecuada, como es la misión del ingeniero industrial [17].

La hipótesis nula y alternativa, son las siguientes:

$\mathbf{H}_{0}$ : La posición y rol del decisor no afectan la eficacia y eficiencia de las decisiones en la solución de las situaciones problemáticas.

$\mathbf{H}_{1}$ : La posición y rol del decisor tienen efecto relevante en la eficacia y eficiencia de las decisiones en la solución de situaciones problemáticas.

La eficacia y eficiencia de las decisiones. Para contrastar la hipótesis se requiere la definición de la eficacia decisional y la eficiencia decisional. La eficacia de la decisión se relaciona con la manera en que el decisor percibe su circunstancia y del lugar jerárquico en que se posiciona y que le permite asumir un rol y un compromiso de cara a los resultados y la expectativa que genera su intervención y decisión. El objetivo es contrastar si el planteamiento que la participación activa y comprometida ante el contexto de actuación, deviene en la mayor eficacia en la elección de opciones.

La eficacia decisional cumple la relación:

$$
E=(C+D) /(A+B+C+D)
$$

que constituye una medida relativa de la proporción de casos factibles de resolver sobre el total de casos planteados, en tanto por uno.

La eficiencia decisional o TES (tiempo estimado de solución) es:

$$
\text { TES }=(T E C+T E D) /(C+D) \text { en horas }
$$

Donde:

TEC: Horas de solución por casos con varias soluciones.

TED: Horas de solución por casos con solución única.

El experimento de decisiones. Bajo la metodología de experimentos [13], al grupo muestral 1 se les presenta diez casos multidimensionales, uno por cada sesión de taller, los que son analizados individualmente y discutidos en grupo acerca de su factibilidad de solución. Cada participante Ilena el cuadro en forma individual acerca de la posibilidad de solución o no, y el tiempo estimado, en caso sea factible resolverlo. La intervención se refiere a que el participante se ubica en el campo 2 productivo y asume una posición sobre la que debe responder con resultados. Cada situación se plantea como una narración, donde el decisor intenta identificar una posible situación problemática que es factible, o no, de resolverse. Se realiza las sesiones de taller. Después de las 10 sesiones posteriores, se comparan los resultados preprueba y posprueba del grupo 1.

Para fines de control se realiza el mismo trabajo con el grupo 2, con el que no se interviene con la identificación de campo y posición sino con la confirmación de su orientación decisional personal básica. Después de las sesiones, se comparan los resultados preprueba y posprueba entre sí y con el grupo 1.

Los resultados se validan mediante estadística inferencial para pequeños grupos donde no se conoce la media ni la desviación estándar, aunque se supone que se comporta con la forma de curva normal [7].

\subsection{Estudio experimental de la eficacia}

\section{$1 .^{\circ}$ Hipótesis}

$H_{0}: \mu_{1}=\mu_{2}$

$\mathrm{H}_{1}: \mu_{1}<\mu_{2}$

Donde:

$\mu_{1}$ : Media de las decisiones planteadas preprueba.

$\mu_{2}$ : Media de las decisiones planteadas posprueba.

Es equivalente a:

$H_{0}: \mu D=0$

$\mathrm{H}_{1}: \mu \mathrm{D}<0$

Donde:

$\mu \mathrm{D}=$ media de las diferencias.

$2 .^{\circ}$ Nivel de significación y nivel de confianza (n.s. y n.c.)

Nivel de significación $\alpha=0.05$

Nivel de confianza $1-\alpha=0.95$

\section{$3 .^{\circ}$ Cálculo de estadísticos para el grupo muestral 1}

En las Tablas 1 y 2 se muestran los estadísticos de muestras relacionadas.

\section{4. ${ }^{\circ}$ Prueba t para muestras relacionadas:}

Se muestra en el Gráfico 1.

5. ${ }^{\circ}$ Resultado sobre hipótesis en el Grupo muestral 1

$\alpha=0.05, \mathrm{gl}=24$

Valor $\mathrm{Tt}=-1.71$ (un lado)

$\mathrm{Tc}=-15.941$

Por lo tanto

Se rechaza la hipótesis básica a un nivel de significación de 0.05 .

Se acepta la hipótesis alternativa. 
Tabla 1. Estadísticos de muestras relacionadas Grupo 1

\begin{tabular}{|cc|c|c|c|c|}
\cline { 2 - 5 } \multicolumn{1}{c|}{} & Media & N & Desviación típ. & $\begin{array}{c}\text { Error típ. de la } \\
\text { media }\end{array}$ \\
\hline \multirow{2}{*}{ Par 1} & $\begin{array}{l}\text { DECIS. PREPRUEBA } \\
\text { DECIS. POSPRUEBA }\end{array}$ & 24,00 & 25 & 8,660 & 1,732 \\
\cline { 2 - 5 } & 69,20 & 25 & 9,967 & 1,993 \\
\hline
\end{tabular}

Fuente: Elaboración propia, empleando SPSS.

Tabla 2. Prueba de muestras relacionadas - Grupo 1

\begin{tabular}{|c|c|c|c|c|c|c|c|c|}
\hline & \multicolumn{5}{|c|}{ Diferencias relacionadas } & \multirow{3}{*}{$\mathrm{t}$} & \multirow{3}{*}{ gl } & \multirow{3}{*}{$\begin{array}{l}\text { Sig. } \\
\text { (bilateral) }\end{array}$} \\
\hline & \multirow{2}{*}{ Media } & \multirow{2}{*}{$\begin{array}{l}\text { Desviación } \\
\text { típ. }\end{array}$} & \multirow{2}{*}{$\begin{array}{l}\text { Error } \\
\text { típ. de la } \\
\text { media }\end{array}$} & \multicolumn{2}{|c|}{$\begin{array}{l}\text { 95\% Intervalo de confianza } \\
\text { para la diferencia }\end{array}$} & & & \\
\hline & & & & Inferior & Superior & & & \\
\hline Par 1 & & & & & & & & \\
\hline $\begin{array}{l}\text { DECIS. PREPRUEBA } \\
\text { DECIS. POSPRUEBA }\end{array}$ & $-45,200$ & 14,177 & 2,835 & $-51,052$ & $-39,348$ & $-15,941$ & 24 & ,000 \\
\hline
\end{tabular}

Fuente: Elaboración propia, empleando SPSS.

Gráfico 1. Aceptación o rechazo de hipótesis- Eficacia de decisiones - Grupo muestral 1.

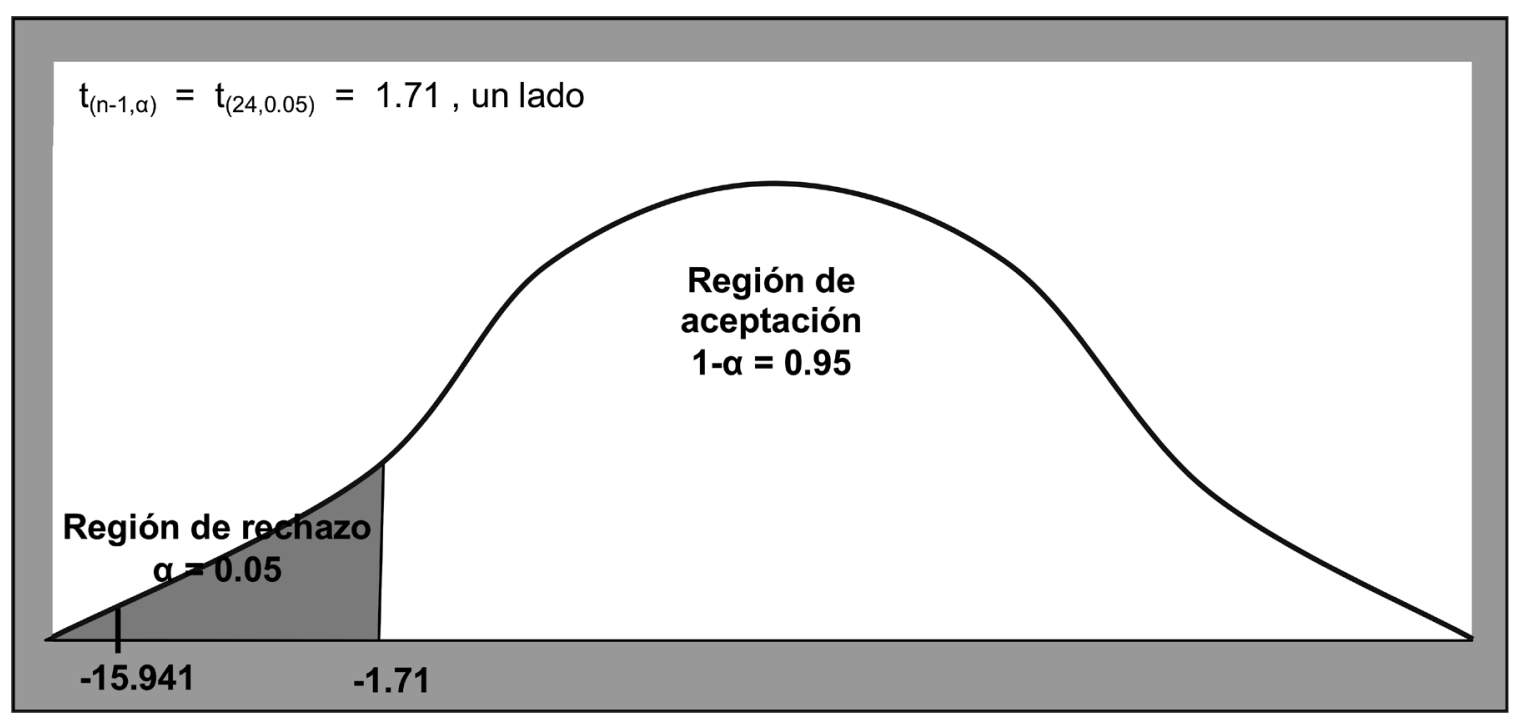

Fuente: Elaboración propia.

\section{6. ${ }^{\circ}$ Cálculo de estadísticos para el Grupo muestral 2}

Para el grupo 2, se muestran los estadísticos de muestras relacionadas en las Tablas 3 y 4 .

7. ${ }^{\circ}$ Prueba t para muestras relacionadas. Se muestra en el gráfico 2. 
Tabla 3. Estadísticos de muestras relacionadas - G 2

\begin{tabular}{|cc|c|c|c|c|}
\cline { 3 - 5 } \multicolumn{1}{c|}{} & Media & $\mathrm{N}$ & Desviación típ. & $\begin{array}{c}\text { Error típ. de la } \\
\text { media }\end{array}$ \\
\hline \multirow{2}{*}{ Par 1} & $\begin{array}{l}\text { DECIS. PREPRUEBA } \\
\text { DECIS. POSPRUEBA }\end{array}$ & 22,00 & 25 & 9,129 & 1,826 \\
\cline { 2 - 5 } & 24,40 & 25 & 7,118 & 1,424 \\
\hline
\end{tabular}

Fuente: Elaboración propia, empleando SPSS.

Tabla 4. Prueba de muestras relacionadas - G 2

\begin{tabular}{|c|c|c|c|c|c|c|c|c|}
\hline & \multicolumn{5}{|c|}{ Diferencias relacionadas } & \multirow{3}{*}{$\mathrm{t}$} & \multirow{3}{*}{$\mathrm{gl}$} & \multirow{3}{*}{$\begin{array}{l}\text { Sig. } \\
\text { (bilateral) }\end{array}$} \\
\hline & \multirow[t]{2}{*}{ Media } & \multirow{2}{*}{$\begin{array}{l}\text { Desviación } \\
\text { típ. }\end{array}$} & \multirow{2}{*}{$\begin{array}{c}\text { Error } \\
\text { típ. de la } \\
\text { media }\end{array}$} & \multicolumn{2}{|c|}{$\begin{array}{l}\text { 95\% Intervalo de confianza } \\
\text { para la diferencia }\end{array}$} & & & \\
\hline & & & & Inferior & Superior & & & \\
\hline Par 1 & & & & & & & & \\
\hline $\begin{array}{l}\text { DECIS. PREPRUEBA } \\
\text { DECIS. POSPRUEBA }\end{array}$ & $-2,400$ & 7,789 & 2,835 & $-5,615$ & ,815 & $-15,541$ & 24 & ,136 \\
\hline
\end{tabular}

Fuente: Elaboración propia, empleando SPSS.

Gráfico 2. Aceptación o rechazo de hipótesis- Eficacia de decisiones - Grupo muestral 2.

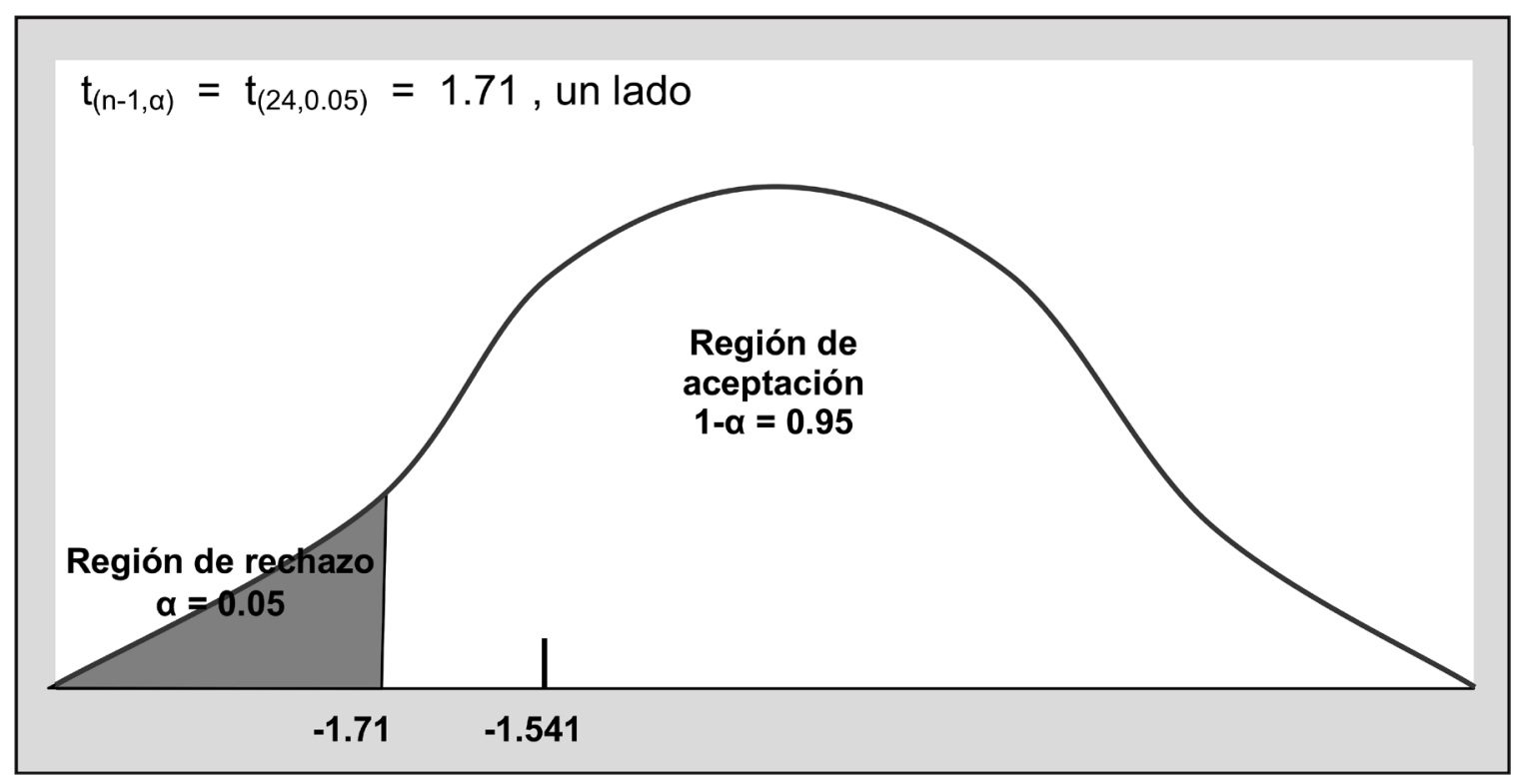

Fuente: Elaboración propia.

\section{8. ${ }^{\circ}$ Resultado sobre hipótesis en el Grupo muestral 2}

$\alpha=0.05, \mathrm{gl}=24$.

Valor $\mathrm{Tt}=-1.71$ (un lado)

$\mathrm{Tc}=-1.541$
Se acepta la hipótesis básica a un nivel de significación de 0.05 .

Se rechaza la hipótesis alternativa. 


\section{Interpretación de los resultados}

Sobre eficacia decisional en el grupo muestral 1. Según la prueba de hipótesis, a un nivel de significación de 0.05 , para la muestra 1 no es posible aceptar la hipótesis de que las elecciones preprueba y las elecciones posprueba tienen igual media. Alternativamente, no se puede rechazar la hipótesis alternativa que indica que las elecciones posprueba son mayores a las decisiones preprueba.

- Se rechaza la hipótesis básica a un nivel de significación de 0.05 .

- Se acepta la hipótesis alternativa un nivel de significación de 0.05 .

Sobre la eficacia decisional en grupo muestral 2. Según la prueba de hipótesis en la muestra 2, a un nivel de significación de 0.05 , se corrobora la hipótesis de que las elecciones preprueba y las elecciones posprueba tienen igual media. Entonces, para esta muestra donde no se incluye la circunstancia, se rechaza la hipótesis alternativa que indica que las elecciones posprueba son diferentes a las decisiones preprueba.

- Se acepta la hipótesis básica a un nivel de significación de 0.05 .

- Se rechaza la hipótesis alternativa un nivel de significación de 0.05 .

Se corrobora que un mayor conocimiento del contexto conlleva a mejores elecciones ante las situaciones problemáticas. Así, el decisor que asume mayor compromiso en el ambiente en que se desenvuelve, está más dispuesto a entender y resolver los problemas.

\subsection{Contenido empírico de la investigación}

\section{Calidad en la elección}

En el grupo 1 se observa que el efecto del contexto, conlleva mejora de la eficacia decisional, o sea, mayor posibilidad de definir problemas y encontrar opciones de solución a las situaciones, además se observa mejora en el tiempo estimado de resolución del problema. En el grupo 2 donde no se incluye la influencia del contexto, la eficacia permanece inalterada y la eficiencia se mantiene en el nivel preprueba. La Tabla 5 es el resumen de los resultados sobre la calidad en la elección.

\section{Influencia del contexto en el grupo muestral 1}

Se observa que, de una posibilidad de resolver el $24 \%$ de los casos, se pasa a la posibilidad de resolver el $69 \%$, donde el perfil decisional cambia drásticamente. El Gráfico 3 muestra la influencia del contexto en el Grupo 1. El sesgo a la izquierda es baja eficacia y el sesgo a la derecha es elevada eficacia decisional.

Complementariamente, los decisores de la muestra 1 estiman que el tiempo empleado en resolver el problema se reduce, de 12 horas en preprueba, disminuye a ocho horas en posprueba, mejorando el indicador de eficiencia, como se muestra en el Gráfico 4.

\section{Comportamiento del decisor del grupo muestral 2}

Se observa que la proporción de resolver los casos en preprueba es de $22 \%$, el cual prácticamente se mantiene en el posprueba (24\%), de manera que el perfil decisional se mantiene, prácticamente, inalterado. El gráfico 5 muestra el comportamiento del decisor, donde no se considera influencia del contexto.

De manera semejante, el tiempo estimado para la resolución de problemas, por los decisores, se ubica en el promedio de 14 horas, lo que se observa en el Gráfico 6.

Tabla 5. Calidad de las decisiones

\begin{tabular}{|c|c|c|c|c|c|c|}
\hline \multirow{2}{*}{$\begin{array}{c}\text { GRUPO } \\
\text { ALEATORIO }\end{array}$} & \multicolumn{3}{|c|}{$\begin{array}{c}\text { EFICACIA } \\
\text { como proporción }\end{array}$} & \multicolumn{3}{|c|}{$\begin{array}{l}\text { EFICIENCIA } \\
\text { en horas }\end{array}$} \\
\hline & Preprueba & Intervención & Posprueba & Preprueba & Intervención & Posprueba \\
\hline Ga1 & 0.24 & $x$ & 0.69 & 12.0 & $x$ & 8.3 \\
\hline Ga2 & 0.22 & -- & 0.24 & 14.4 & -- & 13.5 \\
\hline Ajustado: & & & & & & \\
\hline
\end{tabular}


Gráfico 3. Eficacia de elección - Grupo 1.

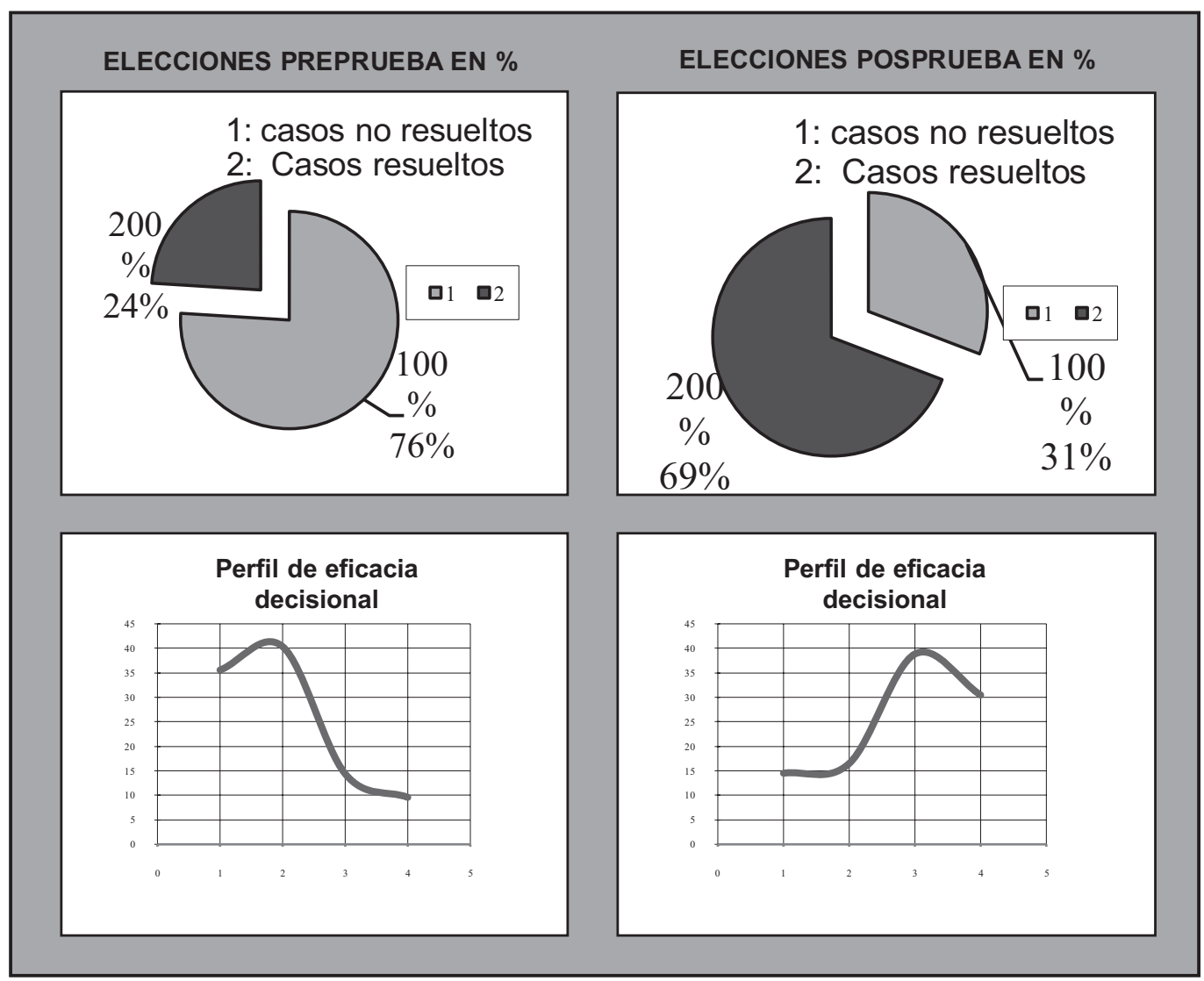

Fuente: Elaboración propia.

Gráfico 4. Eficiencia de elección - Grupo 1.

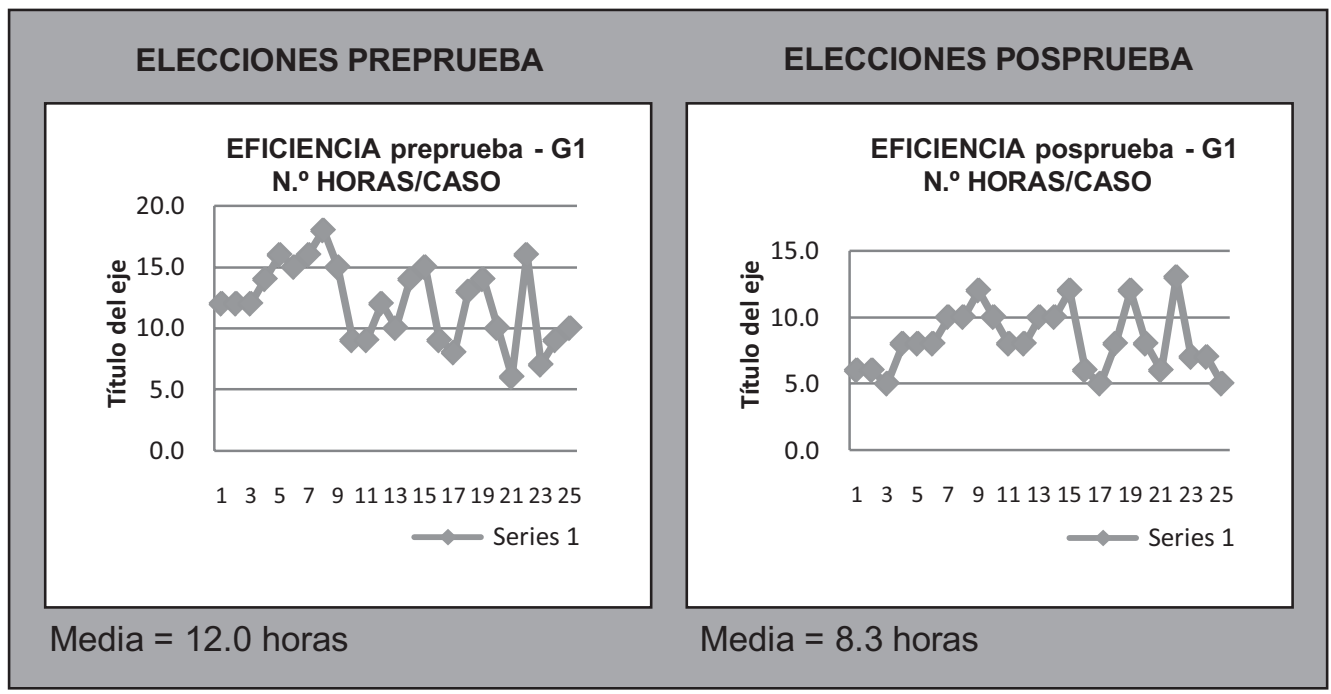

Fuente: Elaboración propia. 
Gráfico 5. Eficacia de elección - Grupo 2.

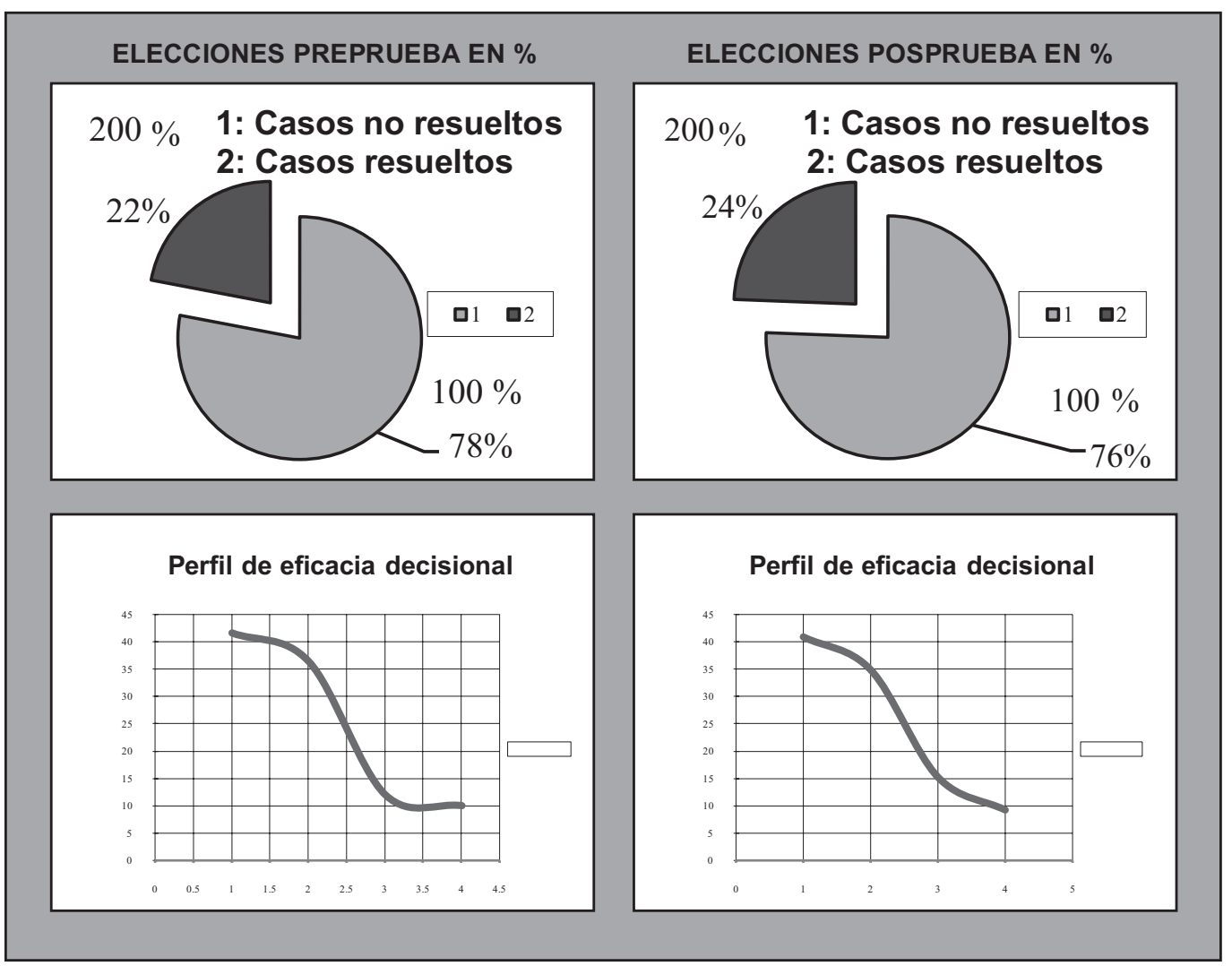

Fuente: Elaboración propia.

Gráfico 6. Eficiencia de elección - Grupo 2.

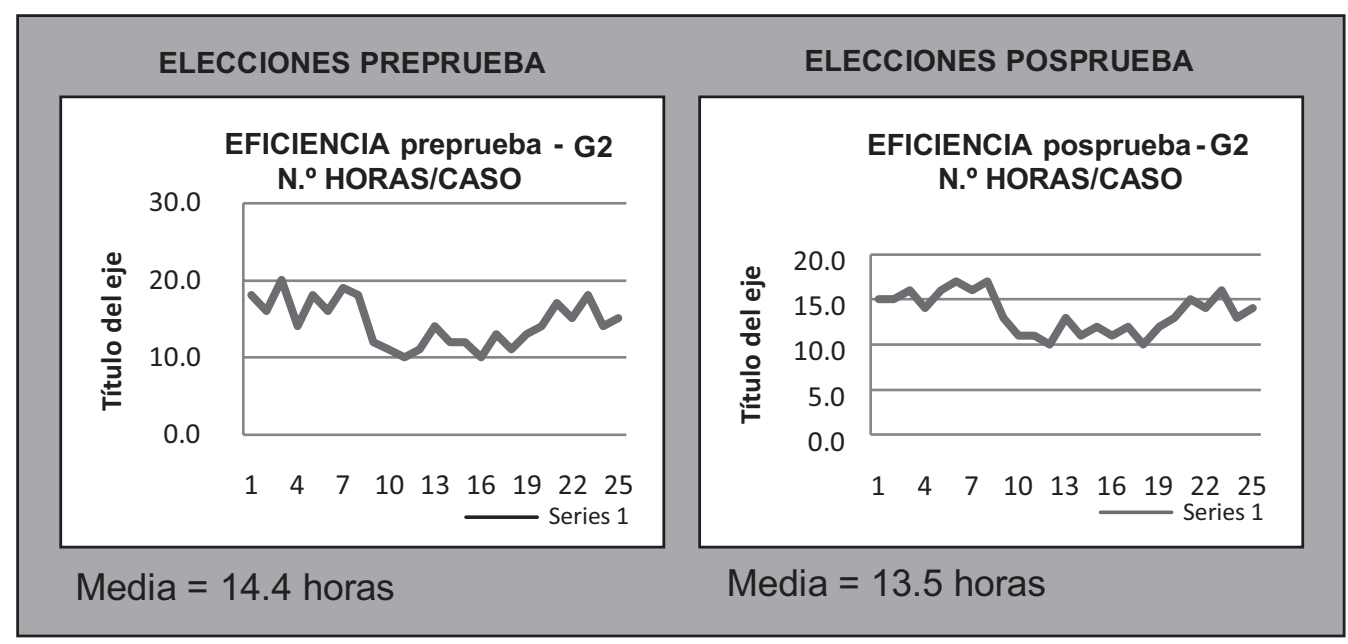

Fuente: Elaboración propia. 


\section{CONCLUSIONES}

De acuerdo a los resultados del estudio, se identifican las siguientes conclusiones:

1. En general, en el grupo con efecto experimental, los resultados preprueba y posprueba, manifiestan variación significativa en el promedio de respuestas y en el resultado individual de los decisores participantes. En el grupo sin influencia experimental, los resultados posprueba presentan respuestas semejantes y resultados similares a los preprueba.

2. La circunstancia influye en la eficacia de las decisiones, lo que tiene efecto en la calidad de las decisiones ya que se enfoca con mayor certeza, aquellas situaciones problemáticas factibles de resolución.

3. El estudio muestra que, si no se incluye la influencia del contexto del problema, se ha de mantener una baja eficacia de las decisiones, medida en un menor porcentaje de casos definidos como solucionables.

4. El decisor ha de mantener una pertinente percepción del contexto y de las situaciones problemáticas, de manera integral y sistémica, a fin de construir una perspectiva holística con conocimiento metodológico del contexto.

5. El decisor ha de tener claramente definida la posición y el rol que le compete dentro de un campo de actuación, ya que esto le ha de permitir una asunción comprometida de las situaciones problemáticas a superar.

6. El decisor ha de desarrollar habilidades metodológicas, de manera que sea posible y previsible entender los efectos y prever las posibles fallas al momento de elegir opciones de solución de problemas.

\section{REFERENCIAS BIBLIOGRÁFICAS}

[1] Acevedo, Adolfo (2011). "Perspectiva y circunstancia en la toma de decisiones: el modelo de las 4D del mundo de la empresa". Industrial Data Revista de Investigación, Vol. 14, N. ${ }^{\circ}$ 2, julio-diciembre 2011. Universidad Nacional Mayor de San Marcos UNMSM.

[2] Acevedo, Adolfo (2010). "El modelo conceptual de las 4 dimensiones para la resolución de problemas". Industrial Data Revista de Investigación, Vol. 13, N. ${ }^{\circ} 2$, julio-diciembre 2010. UNMSM
[3] Acuña, Eduardo (2002). Cuentos del Trabajo en Chile. Editorial Universitaria. Santiago de Chile, Chile.

[4] Ackoff, Russell (2000). Recreación de las corporaciones. Un diseño organizacional para el siglo XXI. Ed. Oxford University Press. México.

[5] Blair \& Whitston (1973). Elementos de Ingeniería de Sistemas Industriales. Editorial Prentice-Hall Internacional. España.

[6] Chiavenato, Idalberto (2004). Introducción a la teoría general de la administración. 7. ${ }^{a}$ ed. Editorial McGraw-Hill. México.

[7] Córdoba, Manuel (2008). Estadística aplicada.

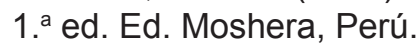

[8] Daft, Richard (2011). Teoría y diseño organizacional. 10. ma ed. CENGAGE Learning Editores, México

[9] Erskine \& Leenders \& Mauffette-Leenders (2001). Writing cases. $4^{\text {th }}$ ed. Richard Ivey School of Business. Ontario, Canada.

[10] Galloway, Jane (2005). "Entrevista con Peter Vaill”. En Desarrollo Organizacional. 6. ${ }^{a}$ ed. Ed. McGraw-Hill, México.

[11] Hernández, Sampieri, Fernández, Baptista (2010). Metodología de la investigación. 5. ${ }^{a}$ ed. Ed. Mcgraw-Hill, Chile.

[12] Lewin, Kurt (2005). "Enfoque de campo: cultura y vida en grupo como procesos cuasiestacionarios". En Desarrollo Organizacional. Transformación y Administración efectiva del cambio. Ed. McGraw-Hill/Interamericana. México.

[13] Martin, David (2008). Psicología experimental. Cómo hacer experimentos en Psicología. 7. ${ }^{\text {a }}$ ed. CENGAGE Learning Editores, México.

[14] Ritchey, T. (2005). "Wicked Problems. Structuring Social Messes with Morphological Analysis". Swedish Morphological Society. www.swemorph.com Extraído el 10-06- 2012.

[15] Vaill, Peter (1974). "La Ingeniería Industrial y los Sistemas Sociotécnicos”. En Groff y Muth, Sistemas de Producción. Edit. El Ateneo, Buenos Aires, Argentina.

[16] Van Gigch, John (1993). Teoría general de sistemas. 3. ${ }^{a}$ reimpr. Ed. Trillas. México.

[17] Zandin, Kjell (2005). Maynard Manual del Ingeniero Industrial. 5. ${ }^{a}$ ed. Editorial McGrawHill, México. 